\title{
Stimulation of Pedicle Screws with Diathermy: Is it a Viable Option to Detect Breaches?
}

\section{Yogesh K Pithwa ${ }^{1 *}$ and Kumar Venkatesh ${ }^{2}$}

${ }^{1}$ FNB Spine Surgery, Consultant Spine Surgeon, HOSMAT Hospital, Bangalore, India

${ }^{2}$ MD Radiology, Chief of Radiology, HOSMAT Hospital, Bangalore, India

\begin{abstract}
Background: To analyse use of monopolar diathermy stimulation of pedicle screws in order to improve breach detection.

Methods: Prospective single-blind study. Patients with preoperative neurological deficit excluded. Pedicle screws inserted freehand into thoracic and lumbar spine. Monopolar diathermy in "cutting" mode at half the strength used for actual cutting purpose was used to stimulate the tap in situ, followed by stimulation of the screw if satisfactory. Elicitation of twitching of local paraspinal muscles and/ or lower limb muscles was considered a positive response, suggestive of pedicle breach. Appropriate revision of screw track was done accordingly. Intraoperative C-arm imaging and postoperative CT scan [blinded evaluation] was done for further verification in all cases. Meticulous clinical assessment was done in the immediate postoperative period to rule out any kind of neurological deterioration.

Results: Seventy-eight consecutive patients undergoing surgical stabilization for varied indications such as trauma [ $n=15]$, spondylolisthesis [n=53], spinal infection $[n=4]$ and spinal deformity [ $n=6]$ were included in the study. Mean age was $42.3+12.3$ years. 468 pedicle screws were inserted. Eleven true positive and three false positive cases were noted. Nine false negative cases were detected on postoperative CT. These breaches were however, $<2 \mathrm{~mm}$ and asymptomatic. Considering this, this method demonstrated sensitivity, specificity, positive predictive value and negative predictive value of $97.63 \%, 100 \%, 100 \%$ and $21.43 \%$, respectively. No patient had any adverse effect related to the diathermy stimulation.
\end{abstract}

Conclusions and Clinical Relevance: Diathermy stimulation of pedicle screws inserted into spine in conjunction with screw track probing and intraoperative $\mathrm{C}$-arm imaging is a viable option to improve accuracy of screw placement, particularly in a setup where no other advanced electrophysiological tests exist.

\section{Level of Evidence: II [diagnostic studies]}

Keywords: Pedicle screw; Breach; Diathermy; Pedicle screw stimulation

\section{Introduction}

Surgical diathermy involves the passage of high frequency alternating electric current through body tissues [1]. Diathermy may be either monopolar or bipolar. In case of monopolar diathermy, high frequency electric current from diathermy machine is delivered to an active electrode held by the surgeon. Density of the electric current is high at the point of contact of diathermy electrode with the body tissue as a result of which a pronounced heating effect occurs locally [1]. Though the heating effect is local, it is the "electric current" that subsequently spreads out in the body and then returns to the diathermy machine via the patient plate electrode (a pad which is kept under the patient) [1].

Direct electrical stimulation of pedicle screws is an accepted modality to assess screw malposition [2,3]. The present study aims to prospectively study the role of direct stimulation of pedicle screws using monopolar diathermy in the "cutting" mode, as an adjuvant modality to assess screw malposition, particularly in a setup which does not have advanced electrophysiological monitors or navigation facilities.

\section{Materials and Methods}

The study was conducted after appropriate approval from the institutional review board. Patients were enrolled after informed and explicit consent as regards the nature and purpose of study and also as regards the potential risk of thermal injury to neural structures.

Patients being considered for surgical stabilization of the thoracic or lumbar spine for varied indications were included in the study. Patients with pre-existing neurological deficit were excluded so as to prevent confounding with any kind of iatrogenic neurological deterioration. Accordingly, seventy-eight patients [thirty males and forty eight females] were included in the study.

Pedicle screws were inserted freehand into thoracic and lumbar spine [4]. No specific restrictions were enforced as regards the type of anaesthetic agents used; inhalational vis-a-vis intravenous [5]. Care was taken, as in all cases to ensure proper application of plate electrode on the patient's body and also to prevent any contact of any other part of patient's body with any metal object so as to prevent diathermy burns. After the initial screw track was made, it was meticulously probed with a ball tipped pedicle sound. If satisfactory, the track was tapped using a tap which was smaller in diameter by $0.5-1 \mathrm{~mm}$ than the final screw to be used [4]. The remaining sequential steps are elaborated in Figure 1 , in the form of a flowchart. While within the screw track, the tap was stimulated with monopolar diathermy [L \& T diathermy, Digital 400, marketed by Johnson \& Johnson] [unlabelled use] in "cutting" mode at half the strength [50 watts] used for actual cutting purposes. The stimulation was done by momentarily touching the diathermy tip to

*Corresponding author: Yogesh K. Pithwa, FNB Spine Surgery, Consultant Spine Surgeon, HOSMAT Hospital, Bangalore, India, Tel: +91-80-26961422 Mobile: +91-9341394471/9880659652; E-mail: info@spine-surgeon.org, yogeshkp1974@gmail.com

Received October 27, 2015; Accepted November 09, 2015; Published November 11, 2015

Citation: Pithwa YK, Venkatesh K (2015) Stimulation of Pedicle Screws with Diathermy: Is it a Viable Option to Detect Breaches? J Spine S6: 006. doi:10.4172/2165-7939.S6-006

Copyright: (c) 2015 Pithwa YK, et al. This is an open-access article distributed under the terms of the Creative Commons Attribution License, which permits unrestricted use, distribution, and reproduction in any medium, provided the original author and source are credited. 


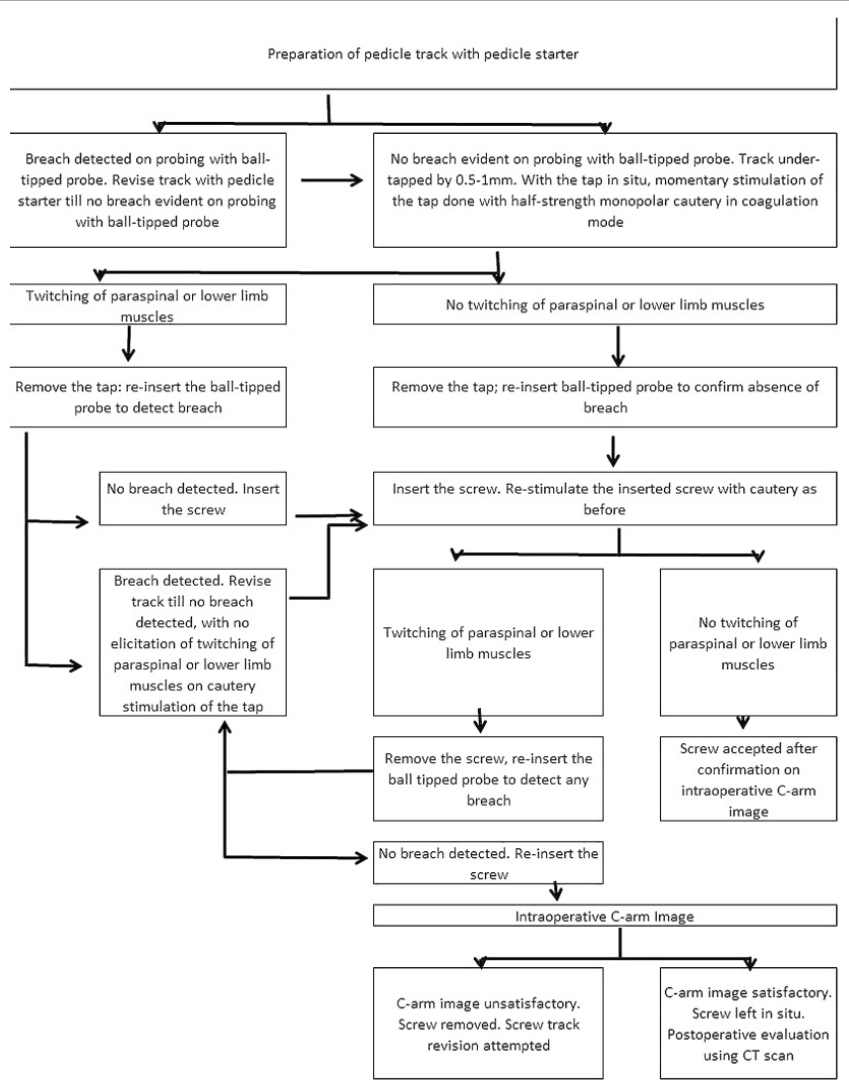

Figure 1: Flowchart of steps followed while using diathermy stimulation of pedicle screws in order to minimize incidence of breaches.

the tap for a fraction of a second and then withdrawing the same so as to minimize the risk of any inadvertent thermal injury to neural structures. Care was taken to avoid direct contact of the tap with any paraspinal muscles so as to prevent direct stimulation of the same. In case of equivocal response, the stimulation was repeated. Elicitation of visible twitching of local paraspinal muscles and/ or palpable twitching of lower limb muscles was termed "positive response", suggestive of pedicle breach which was verified with repeat probing. Visible twitching of paraspinal muscles was considered more pertinent in thoracic levels while palpable twitching of the lower limb muscles was considered more pertinent in lumbosacral levels. Tapped track was probed once again with the pedicle sound so as to feel continuous serrations due to tapping of the bony track. Discontinuity in serrations suggested a pedicle breach and the screw track were revised accordingly. If the operating surgeon was reasonably certain about the accuracy of the prepared track on probing with the pedicle sound, screw was inserted despite there being a positive response on stimulation of the tap. The screw too, was stimulated with monopolar diathermy in the "cutting" mode at half the strength used for actual cutting purposes. Response to this stimulation too, was treated in the same manner as with the tap. Intraoperative $\mathrm{C}$-arm imaging was done in both, anteroposterior and lateral projections for further verification in all cases. Carm image was evaluated for pedicle breach as per the criteria reported by Kim et al. [6]. Accordingly, pedicle screw tips seen crossing the midline on anteroposterior projection were suspected to have a medial breach; while screw tips found lateral to the lateral vertebral body strongly suggested the possibility of lateral breach. If the Carm image revealed sub-optimal screw position in conjunction with a positive response on diathermy stimulation, it was revised otherwise it was left in situ. Pedicle screws with a positive response on diathermy stimulation but without evidence suggestive of breach on probing with the pedicle sound or on the $\mathrm{C}$-arm image were left in situ. Postoperative CT scan was a routine for all cases for further verification of screw position. Wide window (4000 window width and 800 window level) was used to reduce metal artifacts [7]. CT images were evaluated by an independent radiologist blinded to the intervention. Only medial and inferior breaches were considered pertinent to the present study in view of these being in more proximity to neural structures.

Detailed neurological assessment was repeated immediately following the surgery to identify any neurological deterioration. Meticulous care was taken to assess neurology in patients, particularly those who had a true positive diathermy stimulation to assess for any kind of adverse effect to the momentary thermal stimulation.

\section{Statistical Methods}

Statistical analysis was done using statistical software GraphPad Instat, version 3.05. Analysis was done using Fisher's exact test so as to detect sensitivity, specificity and predictive values for this method. Two-tailed $p$-value of $<0.05$ was considered significant.

\section{Results}

Seventy-eight consecutive patients undergoing surgical stabilization for varied indications such as trauma [ $\mathrm{n}=15]$, spondylolisthesis $[\mathrm{n}=53]$, spinal infection $[n=4]$ and spinal deformity $[n=6]$ were included in the study. Mean age was $42.3+12.3$ years.

Four hundred and sixty-eight pedicle screws were inserted in these seventy-eight patients. Of these, 105 screws were in the thoracic spine while 363 were in the lumbosacral spine. All these were titanium pedicle screws. Eleven true positive stimulations, four in thoracic and seven in lumbosacral spine were identified. These were confirmed to be "true" positive on probing the pedicle track with the ball-tipped pedicle sound and/ or assessment of the intraoperative C-arm image. Of these, nine were successfully revised during primary surgery while the other two were abandoned in view of inability to successfully revise those. Three false positive stimulations were noted. These were confirmed to be false positive on repeat probing, intraoperative $\mathrm{C}$-arm imaging and postoperative CT scan. Nine false negative cases with medial breaches [1.92\%] were detected on postoperative CT, five being in the thoracic and four being in the lumbosacral spine (Figure 2). These breaches were however less than $2 \mathrm{~mm}$ and asymptomatic [8]. Considering this, this method demonstrated sensitivity, specificity, positive predictive value and negative predictive value of $97.63 \%, 100 \%, 100 \%$ and $21.43 \%$, respectively.

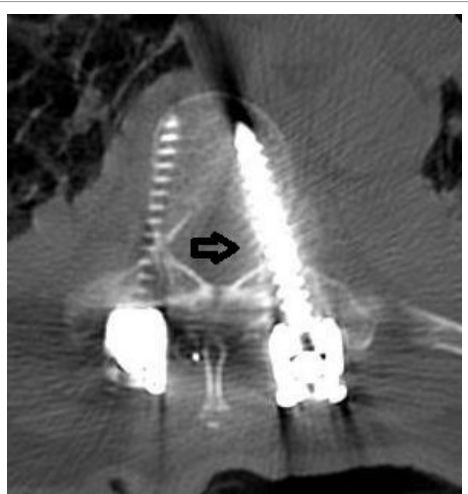

Figure 2: Postoperative CT scan revealing a medial breach $<2 \mathrm{~mm}$, which was false negative on diathermy stimulation and was asymptomatic, as well. 
No patient had neurological deterioration, or any other kind of adverse effect related to the stimulation.

\section{Discussion}

Insertion of pedicle screws has become a popular procedure for stabilizing the thoracolumbar spine. Three-column fixation offered by pedicle screws cannot be emulated by any other form of contemporary fixation. Initial reservation related to use of thoracic pedicle screws has also gradually faded away with an increasing number of surgeons opting to use these over other methods of anchoring the thoracic spine [9].

With an increasing use of pedicle screws, concerns related to pedicle breach have led to the development of numerous modalities during surgery to minimize the same. Numerous checks ranging from basic ones such as probing the pedicle screw track with pedicle sound and intraoperative $\mathrm{C}$-arm imaging to sophisticated modalities such as navigation-assisted screw insertion, iso-C 3-dimensional fluoroscopy imaging, and electrophysiological tests including SSEP, MEP and EMG tracing have been employed to decrease incidence of pedicle screw breach $[2,6,10,11]$. Among all these modalities, SSEP is the only realtime monitoring. Though availability of these sophisticated modalities may not be an issue in developed countries, availability across the globe is extremely limited.

No single method of checking pedicle violation is absolutely accurate [12]. An almost universally practised method of probing the pedicle tract with a ball-tipped probe has been shown to have a relatively poor accuracy, with medial breaches as high as upto $32 \%$ being missed out on the same [13]. Hence a combination of methods would yield a cumulative outcome of reasonably high accuracy and hence, safety.

In a cadaveric study of analysis of "free-hand" screw insertion in thoracic spine, $\mathrm{Xu}$ et al. noted $54.7 \%$ "miss" rates [14]. Additional usage of fluoroscopy by Weinstein et al. in another cadaveric study of pedicle screw insertion from T11-S1 decreased the overall pedicle "miss" rate to $21 \%$ [15]. Further additional usage of pedicle probing with a flexible ball-tipped probe decreased the medial and inferior wall violation to $2 \%$ [thirteen of 640 screws] in a study by Karapinar et al. [16]. In an analysis of 3204 thoracic pedicle screws inserted with "free-hand" technique with adjuvant pedicle probing, fluoroscopy and neurophysiologic monitoring, only $1.7 \%$ incidence of asymptomatic medial wall violation was noted [4]. The present study of "free-hand" insertion in thoracolumbar spine with adjuvant pedicle probing, fluoroscopy and thermal stimulation of pedicle screws with monopolar electrocautery gave a similar outcome of only $1.92 \%$ medial violation which was also asymptomatic.

Though inhalational anaesthesia agents are known to hamper electrophysiological monitoring, these agents are unlikely to hamper triggered EMG testing by direct stimulation of pedicle screws [5]. Since the present study involved similar direct screw stimulation by diathermy, no specific restriction in terms of anaesthesia agents was found necessary during surgery.

Though there was a $0.88 \%$ [three of 342] incidence of false positive cases using diathermy stimulation, no symptomatic false negative case with medial breach was noted using this method giving it $100 \%$ specificity in this regard. A great number of false positive cases can be reduced in this method by avoiding direct contact of the tap or screw with paraspinal muscles.

An obvious limitation of this study as well as this method is lack of standardization of the stimulation. Though an arbitrary standard of half the strength used for routine cutting was adopted for this study, it would be certainly advantageous to get some form of standardization in future studies. Additionally, variability in osteoporotic pedicles and paediatric pedicles is a factor which could not be addressed in the current study. However, even triggered EMG testing of pedicle screws is known to have variable "cut-offs" depending on the severity and duration of compression of involved nerves [17]. Another obvious limitation of this method is the lack of real-time monitoring. Another one of the major concerns is the potential risk of thermal injury to neural structures. Theoretically however, literature exists on thermal stimulation too of neural structures; Plaghki et al. having reported stimulation of peripheral nerves using non-noxious radiant heat produced by laser [18]. Literature exists on use of monopolar diathermy for making surgical skin incisions too, without causing any thermal necrosis [1] None the less, though a concern for thermal injury does exist; no patient in the present study had any evidence of such a complication. By reducing the contact time of diathermy with tap or screw to a fraction of a second, this potential risk can certainly be mitigated as evinced in this study.

Though electrophysiological monitoring and additional usage of navigation systems, 3-dimensional CT scanning have been documented to supplement surgical skills and improve accuracy in pedicle screw insertion; these sophisticated equipment's are not universally available in all places. Hence, usage of a simple monopolar diathermy which is universally available in all operation rooms to improve pedicle screw insertion would be definitely advantageous. To the best of authors' knowledge, the present study is the first one to analyse the efficacy of this method in a scientific manner. This method is not being envisaged as a substitute for electrophysiological monitoring; but can certainly be an option to exercise when the surgeon is doubtful of the pedicle screw and does not have access to advanced systems such as electrophysiological monitoring.

\section{References}

1. Vedbhushan ST, Mulla MA Haroonrasid Chandrashekhar DM (2013) Surgical incision by high frequency cautery. Indian J Surg 75: 440-443.

2. Sutter M, Eggspuehler A, Muller A, Dvorak J (2007) Multimodal intraoperative monitoring: an overview and proposal of methodology based on 017 cases. Eur Spine J 16 Suppl 2: S153-161.

3. Reidy DP, Houlden D, Nolan PC, Kim M, Finkelstein JA (2001) Evaluation of electromyographic monitoring during insertion of thoracic pedicle screws. J Bone Joint Surg Br 83: 1009-1014.

4. Kim YJ, Lenke LG, Bridwell KH, Cho YS, Riew KD (2004) Free Hand Pedicle Screw Placement in the Thoracic Spine: Is it Safe? Spine 29: 333-342.

5. Chen Z (2004) The effects of isoflurane and propofol on intraoperative neurophysiological monitoring during spinal surgery. J Clin Monit Comput 18 : 303-308.

6. Kim YJ, Lenke LG, Cheh G, Riew KD (2005) Evaluation of pedicle screw placement in the deformed spine using intraoperative plain radiographs: a comparison with computerized tomography. Spine (Phila Pa 1976) 30: 2084 2088.

7. Stradiotti P, Curti A, Castellazzi G, Zerbi A (2009) Metal-related artifacts in instrumented spine. Techniques for reducing artifacts in CT and MRI: state of the art. Eur Spine J 18 Suppl 1: 102-108.

8. Modi HN, Suh SW, Fernandez H, Yang JH, Song HR (2008) Accuracy and safety of pedicle screw placement in neuromuscular scoliosis with free-hand technique. Eur Spine J 17: 1686-1696.

9. Zeiller SC, Lee J, Lim M, Vaccaro AR (2005) Posterior thoracic segmental pedicle screw instrumentation: evolving methods of safe and effective placement. Neurol India 53: 458-465.

10. Boachie-Adjei O, Girardi FP, Bansal M, Rawlins BA (2000) Safety and efficacy of pedicle screw placement for adult spinal deformity with a pedicle-probing conventional anatomic technique. J Spinal Disord 13: 496-500. 
11. Rajasekaran S, Vidyadhara S, Ramesh P, Shetty AP (2007) Randomized clinical study to compare the accuracy of navigated and non-navigated thoracic pedicle screws in deformity correction surgeries. Spine (Phila Pa 1976) 32 E56-64.

12. Mattei TA, Meneses MS, Milano JB, Ramina R (2009) "Free-hand" technique for thoracolumbar pedicle screw instrumentation: critical appraisal of current "state-of-art". Neurol India 57: 715-721.

13. Sedory DM, Crawford JJ, Topp RF (2011) The reliability of the ball-tipped probe for detecting pedicle screw tract violations prior to instrumenting the thoracic and lumbar spine. Spine (Phila Pa 1976) 36: E447-453.

14. Xu R, Ebraheim NA, Ou Y, Yeasting RA (1998) Anatomic considerations of pedicle screw placement in the thoracic spine. Roy-Camille technique versus open-lamina technique. Spine (Phila Pa 1976) 23: 1065-1068.
15. Weinstein JN, Spratt KF, Spengler D, Brick C, Reid S (1988) Spinal pedicle fixation: reliability and validity of roentgenogram-based assessment and surgical factors on successful screw placement. Spine (Phila Pa 1976) 13 1012-1018.

16. Karapinar L, Erel N, Ozturk H, Altay T, Kaya A (2008) Pedicle screw placement with a free hand technique in thoracolumbar spine: is it safe? J Spinal Disord Tech 21: 63-67.

17. Holland NR, Lukaczyk TA, Riley LH 3rd, Kostuik JP (1998) Higher electrical stimulus intensities are required to activate chronically compressed nerve roots. Implications for intraoperative electromyographic pedicle screw testing. Spine (Phila Pa 1976) 23: 224-227

18. Plaghki L, Bragard D, Le Bars D, Willer JC, Godfraind JM (1998) Facilitation of a nociceptive flexion reflex in man by nonnoxious radiant heat produced by a laser. J Neurophysiol 79: 2557-2567.
This article was originally published in a special issue, Advanced Techniques in Spine Surgery handled by Editor(s). Dr. Alessandro Landi, University of Rome, Italy 